\title{
Synthesis of Mesoporous Titania by Using Alkoxide Precursor and CTAB as Template
}

S. E. Borjas-García ${ }^{1}$, Padma Sahare ${ }^{2}$. N. Dasgupta-Schubert ${ }^{2}$, P. Martínez-Torres ${ }^{1}$, D. K. Tiwari ${ }^{3}$, A. Medina-Flores ${ }^{4}$, G. Rosas ${ }^{4}$

${ }^{1}$ Instituto de Física y Matemáticas, UMSNH, Morelia, Michoacán, México.

${ }^{2}$ Facultad de Ciencias Físico-Matemáticas, UMSNH, Morelia, Michoacán, México.

${ }^{3}$ CONACyT-El Colegio de Michoacán, La Piedad, Michoacán, México.

${ }^{4}$ Instituto de Investigación en Metalurgia y Materiales, UMSNH, Morelia, Michoacán, México.

Crystalline $\mathrm{TiO}_{2}$ is a very attractive ceramic oxide because it shows excellent performance in photocatalytic reactions and in energy conversion [1]. A mesoporous material like mesoporous titania is one that has pores in a range between 2 and $50 \mathrm{~nm}$. Also, this kind of material has attracted much attention because of its increased surface area. At present, there are several kinds of mesoporous ceramic metal oxides that have been synthesized by different template methods like "Soft template method" and "hard template method" [2]. In the case of the synthesis of mesopores of titania, many procedures have been developed using titanium salts as precursor [2-6]. However, there is not enough research about the synthesis of mesopores of titania with alkoxide precursors using CTAB (hexadecyltrimethyl ammonium bromide) as template. In this work, mesoporous titania was synthesized by using a combination of the sol-gel and soft hydrothermal methods. The mesoporous titania of this work was prepared by using titanium butoxide (Aldrich), triethanolamine,TEAOH (J. T. Baker), CTAB (Sigma), sodium hydroxide (J. T. Baker) as Ti-source, hydrolysis stabilizer, template and alkaline material, respectively. In the first step, a stock Ti-solution was prepared by mixing $0.25 \mathrm{~mol}$ of TEAOH with $0.125 \mathrm{~mol}$ of Ti-source with vigorous stirring for one day. After that, two additional solutions were prepared. The first one was obtained by dissolving $3.1935 \mathrm{~g}$ of stock Ti-solution and $0.9112 \mathrm{~g}$ of CTAB in $10 \mathrm{~g}$ of distilled water. For the second solution, $1.6 \mathrm{~g}$ of $\mathrm{NaOH}$ was dissolved in $1.8015 \mathrm{~g}$ of distilled water. In a second step, the Na-solution was added slowly (drop by drop) to Ti-CTAB-solution and stirred. The final material obtained was stirred and heated in a hot plate with stirring at $40{ }^{\circ} \mathrm{C}$ for one day in order to evaporate the excess water. This treatment was done in an open glass flask. The gel obtained was aged in a closed glass flask at $80{ }^{\circ} \mathrm{C}$ for 1 day. After the hydrothermal treatment, the sample was washed with $100 \mathrm{ml}$ of distilled water and centrifuged three times at $12000 \mathrm{rpm}$. Then, the material was dried at $80{ }^{\circ} \mathrm{C}$ for 1 day. Furthermore, it was necessary to calcine the sample at $560{ }^{\circ} \mathrm{C}$ for $1 \mathrm{~h}$ to eliminate the residual organic template. In order to observe the effect of temperature ageing in the synthesis procedure, another sample was prepared at $100{ }^{\circ} \mathrm{C}$. The morphology of as-synthesized sample was corroborated using a high resolution scanning electron microscope (HR-SEM, Jeol JSM-7800F).

Figure 1 and 2 show images of as-synthesized mesoporous titania samples at 80 and $100^{\circ} \mathrm{C}$, respectively. The size of mesopores in these samples was in the range of 10 and $50 \mathrm{~nm}$. There are several parameters of synthesis that allow shaping the mesopores in titania; for example, the amount of water, the temperature of the hydrothermal treatment, the amount of mineralized material, the source of titanium and the amount of template material. According to our results, the critical synthesis conditions are the molar ratio between the template and titanium and, the concentration of water. Also, the kind of alkaline material and its amount during the synthesis allows controlling the $\mathrm{pH}$ that could be a critical factor to obtain mesoporous titania. It is important to mention that we stabilized titanium butoxide with 
TEAOH in order to make the gel formation slower and to increase the possibility of the formation of mesopores.

References:

[1] X. Chen and S. S. Mao, Chem. Rev. 107 (2007) p. 2891.

[2] D.W. Bruce, D. O'Hare and R.I. Walton, "Porous materials", 1st ed. (Wiley, United Kingdom, 2010) p. 1.

[3] P. Yang et al, Nature 396 (1998) p. 152.

[4] P. Yang et al, Chem. Mater. 11 (1999) p. 2813.

[5] E. L. Crepaldi et al, New J. Chem. 27 (2003) p. 9.

[6] J. Lee et al, Nat. Mater. 7 (2008) p. 222.

[7] The authors acknowledge funding from H. Consejo Técnico of Instituto de Física y Matemáticas, and Consejo Nacional de Ciencia y Tecnología (CONACyT), México.
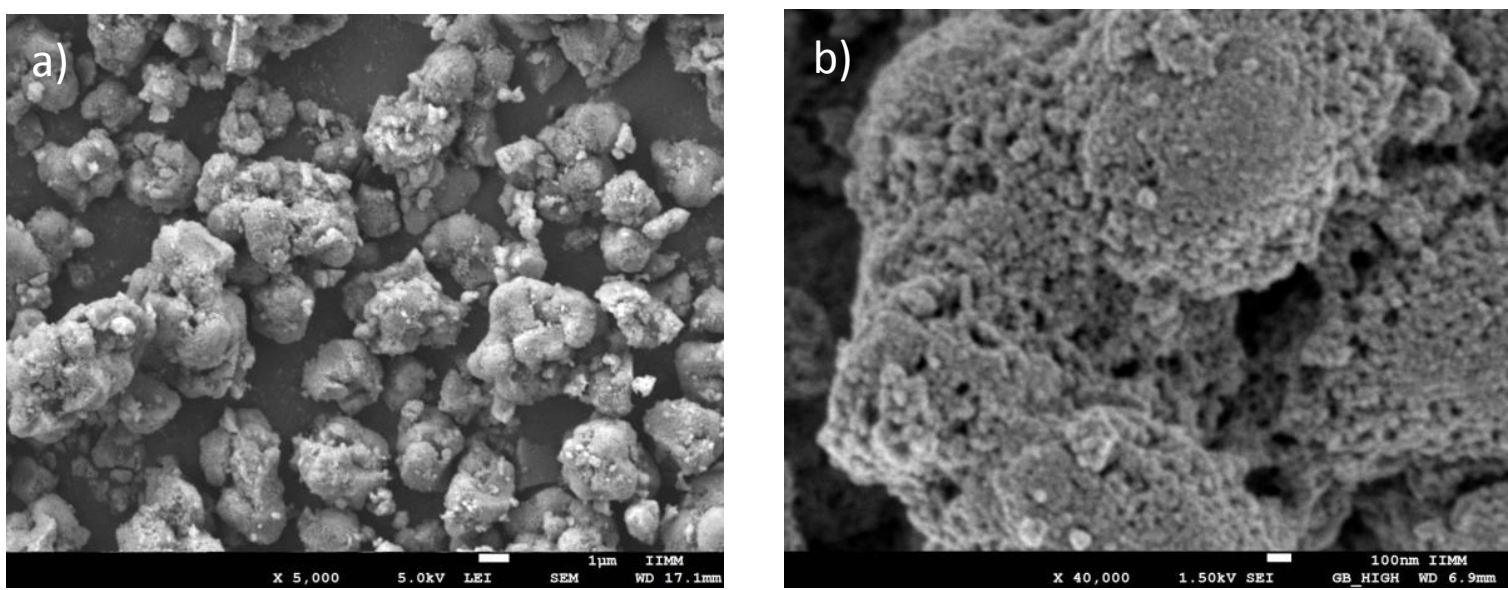

Figure 1. SEM images of as-synthesized mesoporous titania at $80{ }^{\circ} \mathrm{C}$ at a) $5000 \mathrm{X}$ and b) $40000 \mathrm{X}$ of magnification.
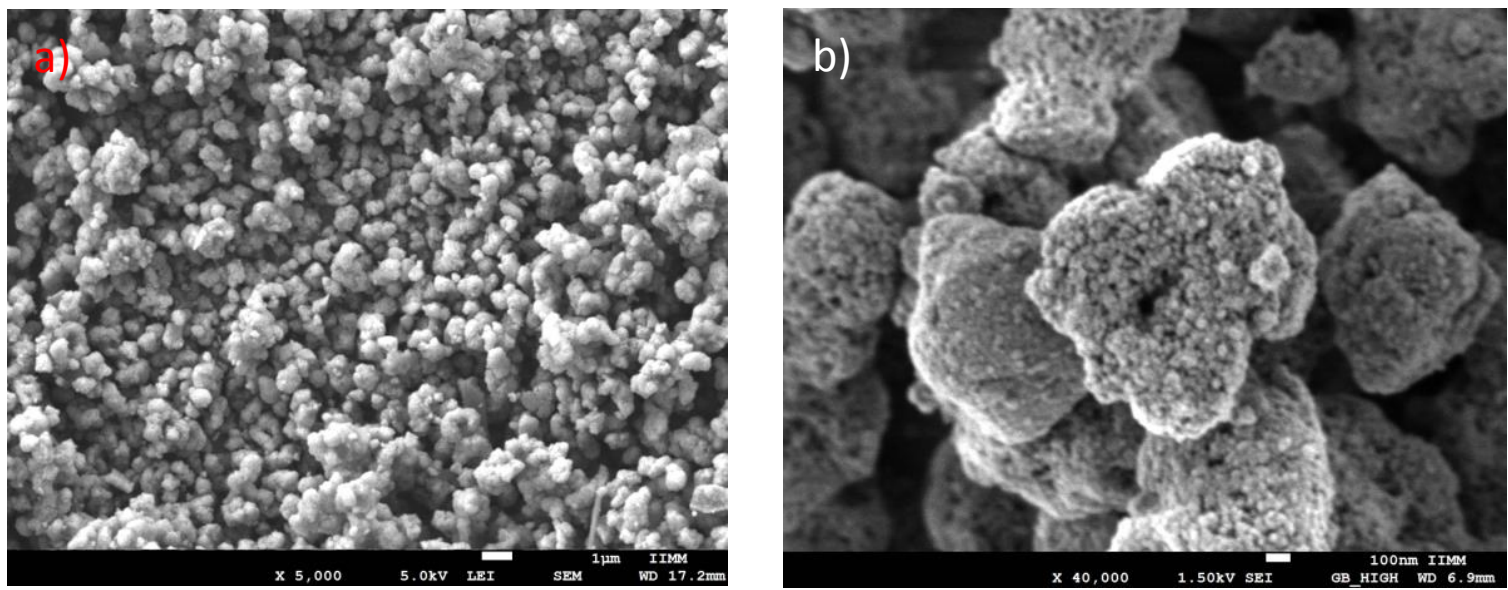

Figure 2. SEM images of as-synthesized mesoporous titania at $100{ }^{\circ} \mathrm{C}$ at a) $5000 \mathrm{X}$ and b) $40000 \mathrm{X}$ of magnification. 